analysis, the following were all associated with serious infection $(p<0.01)$ : splenectomy, use of cyclophosphamide, mycophenolate and rituximab, which is shown in figure 1 and figure 2 .

Conclusions In the largest observational European Registry of SLE patients, one third of the jSLE patients suffered serious infections. Higher SLEDAI score, renal involvement and immunosupressant and corticosteroids use were independent associated factors to the presence of serious infection in jSLE, as well as smoking.

Funding Source(s): FIS Grant PI11/02857 (Instituto Carlos III, Fondos FEDER) has supported this work.

\section{IMMODULATORY MEDICATION USE FOR YOUTH WITH NEWLY-DIAGNOSED SYSTEMIC LUPUS ERYTHEMATOSUS}

${ }^{1}$ Alaina Davis, ${ }^{2}$ Andrea M Knight*, ${ }^{3}$ Marisa Klein-Gitelman, ${ }^{4}$ Jennifer Faerber, ${ }^{4}$ Hannah Katcoff, ${ }^{5}$ Zuleyha Cidav, ${ }^{5}$ David Mandell. ${ }^{1}$ Monroe Carell Junior Children's Hospital at Vanderbilt; ${ }^{2}$ The Hospital for Sick Children, Toronto; ${ }^{3}$ Ann and Robert $H$ Lurie Childrens Hospital of Chicago; ${ }^{4}$ The Children's Hospital of Philadelphia; ${ }^{5}$ University of Pennsylvania

\subsection{6/lupus-2019-Ism.182}

Background To examine immunomodulatory medication use for youth with systemic lupus erythematosus (SLE) during their first year of care.

Methods We conducted a retrospective cohort study using deidentified administrative claims for 2000 to 2013 from Optum $\left(\right.$ C Clinformatics ${ }^{\circledR}$ DataMart for youth ages $10-24$ years with an incident diagnosis of SLE (3 International Classication of Diseases, Ninth Revision codes for SLE 710.0, each $>30$ days apart). We determined the proportion of subjects filling a prescription for an immunomodulatory mediation, defined as hydroxychloroquine or an immunosuppressant (excluding glucocorticoids), within 3, 6, and 12 months after the first SLE diagnosis code (index date). We used a Cox proportional hazards regression model to examine associations between time to immunomodulatory prescription fill within 12 months and demographic and disease factors (age, race/ethnicity, household education level, region, history of seizures/stroke, history nephritis).

Results We identified 650 youth with an incident diagnosis of SLE. In the 12 months following the index date, 511 (79\%) of youth had a prescription fill for an immunomodulatory medication. For those with a prescription fill for hydroxychloroquine in the first year $(n=457,70 \%), 374(58 \%)$ and $407(63 \%)$ of youth filled the medication within 3 months and 6 months from the index date, respectively (table). For those with a prescription fill for an immunosuppressant $(n=221,34 \%)$ in the first year, $114(18 \%)$ and $162(25 \%)$ of youth filled the medication within 3 months and 6 months from the index date, respectively (Table). Location in the Northeast region was significantly associated with a longer time to immunomodulatory prescription fill within 12 months, compared to location in the South $(\mathrm{HR}=0.686$, 95\% CI 0.50 0.94). There were no statistically significant associations for the other demographic and disease factors.

Conclusions Among youth with newly-diagnosed SLE, hydroxychloroquine use is prevalent although not universal, and immunosuppressant use is notably low during the first year of care. As poorly controlled SLE disease activity can lead to organ damage, further work is needed to identify potential
Abstract 182 Table 1 Immunomodulatory Medication Use in Youth with Newly-Diagnosed SLE, N=650

\begin{tabular}{lccc}
\hline $\begin{array}{l}\text { Proportion with prescription } \\
\text { fills after first SLE diagnosis code, } \\
\mathbf{n}(\%)\end{array}$ & $\begin{array}{c}\text { Within } \\
3 \text { months }\end{array}$ & $\begin{array}{c}\text { Within } \\
6 \text { months }\end{array}$ & $\begin{array}{c}\text { Within } \\
1 \text { year }\end{array}$ \\
\hline $\begin{array}{l}\text { Immunomodulatory medication } \\
\text { (hydroxychloroquine or }\end{array}$ & $428(66)$ & $460(71)$ & $511(78)$ \\
$\begin{array}{l}\text { immunosuppressant) } \\
\text { Hydroxychloroquine }\end{array}$ & $374(58)$ & $407(63)$ & $457(70)$ \\
Immunosuppressant & $114(18)$ & $162(25)$ & $221(34)$
\end{tabular}

Immunosuppressant medications include: mycophenolate mofetil, azathioprine, leflunomide, methotrexate, tacrolimus, and oral cyclophosphamide.

factors contributing to suboptimal immunomodulatory medication use in this population.

Funding Source(s): The Childhood Arthritis and Rheumatology Research Alliance, Alpha Omicron Pi Foundation

\section{A PHASE 1B/2A TRIAL OF TOFACITINIB, AN ORAL JANUS KINASE INHIBITOR, IN SYSTEMIC LUPUS ERYTHEMATOSUS}

${ }^{1}$ Sarfaraz Hasni*, ${ }^{2}$ Sarthak Gupta, ${ }^{3}$ Michael A Davis, ${ }^{4}$ Elaine Poncio, ${ }^{5}$ Yenealem TemesgenOyelakin, ${ }^{6}$ Ann Biehl, ${ }^{7}$ Philip Carlucci, ${ }^{5}$ Xinghao Wang, ${ }^{8}$ Isabel Ochoa-Navas, ${ }^{8}$ Zerai G Manna, ${ }^{2}$ Mohammad Naqi, ${ }^{9}$ Yinghui Shi, ${ }^{10}$ Donald E Thomas, ${ }^{11}$ Jinguo Chen, ${ }^{12}$ Angelique Biancotto, ${ }^{13}$ Richard Apps, ${ }^{14}$ Foo Cheung, ${ }^{15}$ Yuri Kotiliarov, ${ }^{16}$ Ashley Babyak, ${ }^{17}$ Katie Stagliano, ${ }^{18}$ Wanxia Tsai, ${ }^{9}$ Laura Vian, ${ }^{19}$ Nathalia R Gazaniga, ${ }^{20}$ Valentina Giudice, ${ }^{21}$ Martin Playford, ${ }^{2}$ Stephen Brooks, ${ }^{5}$ Rishi R Goel, ${ }^{22}$ Meggan MacKay, ${ }^{23}$ Peter Gregersen, ${ }^{22}$ Betty Diamond, ${ }^{24}$ Xiaobai Li, ${ }^{25}$ Alan Remaley, ${ }^{26}$ Nehal Mehta, ${ }^{8}$ John O'Shea ${ }^{21}$ Massimo Gadina, ${ }^{27}$ Mariana J Kaplan. ${ }^{1}$ The Office of Clinical Director, National Institute of Arthritis and Musculoskeletal and Skin Diseases, National Institutes of Health; ${ }^{2}$ NIAMS/NIH; ${ }^{3}$ National Institute of Arthritis and Musculoskelatal and Skin Diseases, NIH; ${ }^{4}$ National Institute of Arthritis and Musculoskeletal and Skin Diseases, National Institutes of Health; ${ }^{5}$ NIH/NIAMS; ${ }^{6}$ National Institute of Arthritis and Musculoskeletal and Skin Diseases; ${ }^{7}$ New York University School of Medicine; ${ }^{8}$ NIAMS; ${ }^{9} \mathrm{NIH} ;{ }^{10}$ Arthritis and Pain Associates of PG County; ${ }^{11}$ NIAID; ${ }^{12}$ Sanofi; ${ }^{13}$ NIH Center for Human Immunology; ${ }^{14}$ The National Institutes of Health; ${ }^{15} \mathrm{CHI} / \mathrm{NIAID} / \mathrm{NIH} ;{ }^{16}$ National Institutes of Health, Clinical Center; ${ }^{17}$ Center for Human Immunology, NIH; ${ }^{18}$ National Institue of Arthritis and Musculoskleatal and Skin Diseases; ${ }^{19}$ The Scripps Research Institute; ${ }^{20}$ University of Salerno; ${ }^{21}$ National Institutes of Health; ${ }^{22}$ Feinstein Institute; ${ }^{23}$ Feinstein Institute For Medical Research; ${ }^{24}$ Clinical Center, $\mathrm{NIH} ;{ }^{25} \mathrm{NHLBI} ;{ }^{26}$ National Heart, Lung and Blood Institute; ${ }^{27}$ NIH NIAMS

\subsection{6/lupus-2019-Ism.183}

Background A pharmacologic intervention that modulates JAK/ STAT signaling pathways represents a novel approach for the treatment of Systemic Lupus Erythematosus (SLE). In animal models of SLE, tofacitinib improved clinical features, immune dysregulation and vascular dysfunction. The STAT4 risk allele is associated with higher risk of severe manifestations in SLE. We hypothesized that immune modulation in response to JAK/ STAT inhibition would be more robust in SLE subjects that carry the STAT4 risk allele.

Methods We conducted a phase $1 \mathrm{~b} / 2 \mathrm{a}$ randomized, doubleblind, placebo-controlled clinical trial of oral tofacitinib, $5 \mathrm{mg}$ twice daily, in 30 SLE subjects (2:1 drug to placebo ratio) with mild to moderate disease activity, stratified by the presence or absence of STAT4 risk allele. Study duration was 84 days (56 days of active treatment ; 28 days of off drug). In addition to recording adverse events (AEs), lipoprotein profile, non-invasive vascular function studies, immuno-phenotyping, and gene expression studies were performed. 